HETEROCYCLES, Vol. 76, No. 1, 2008, pp. -. (c) The Japan Institute of Heterocyclic Chemistry

Received, 1st April, 2008, Accepted, 1st May, 2008, Published online, 8th May, 2008. COM-08-S(N)85

\title{
SYNTHESIS AND REACTIVITY OF
}

\section{3-METHYLSULFINYL-2H-CYCLOHEPTA[b]FURAN-2-ONES}

\section{Junya Higashi, ${ }^{a}$ Kazuyuki Okada, ${ }^{\text {a }}$ Taku Shoji, ${ }^{a}$ Kozo Toyota, ${ }^{a}$ Masataka Watanabe, ${ }^{\mathrm{b}}$ Masafumi Yasunami, ${ }^{\mathrm{c}}$ Shigeru Kikuchi, ${ }^{\mathrm{d}}$ Shunji Ito, ${ }^{\mathrm{e}}$ and Noboru Morita* ${ }^{\text {a† }}$}

${ }^{\mathrm{a}}$ Department of Chemistry, Graduate School of Science, Tohoku University, Sendai, 980-8578, Japan

nmorita@m.tains.tohoku.ac.jp

${ }^{\mathrm{b}}$ Institute of Multidisciplinary Research for Advanced Materials, Tohoku University, Sendai 980-8577, Japan

${ }^{c}$ Department of Materials Chemistry and Engineering, College of Engineering, Nihon University, Koriyama, 963-8642, Japan

${ }^{\mathrm{d}}$ Graduate School of Science and Technology, Kumamoto University, Kumamoto 860-8555, Japan

${ }^{\mathrm{e}}$ Graduate School of Science and Technology, Hirosaki University, Hirosaki 036-8561, Japan

\begin{abstract}
H-Cyclohepta[b]furan-2-ones (2a,b) reacted with dimethyl sulfide dittiflate to give dimethyl(2-oxo-2H-cyclohepta[b]furan-3-yl)sulfonium trifluoromethanesulfonates (3a,b), which were treated with $\mathrm{Et}_{3} \mathrm{~N}$ to give 3-methylthio-2H-cyclohepta[b]furan-2-ones (4a,b). Sulfides $\mathbf{4 a}$ and $\mathbf{4 b}$ were oxidized with $m$-CPBA to give corresponding sulfoxides $(\mathbf{5 a}, \mathbf{b})$ and sulfones $(\mathbf{6 a}, \mathbf{b})$. The sulfoxides $(\mathbf{5 a}, \mathbf{b})$ thermally underwent coupling reaction to give 3,3'-bi-2H-cyclohepta[b]furan-2-ones $(\mathbf{7 a}, \mathbf{b})$. The sulfoxides $(\mathbf{5 a}, \mathbf{b})$ reacted with trifluoromethanesulfonic anhydride $\left(\mathrm{Tf}_{2} \mathrm{O}\right)$ to afford sulfonium ions $\mathbf{8 a}$ and $\mathbf{8 b}$ at lower temperature, which reacted with $\mathbf{2 a}$ or $\mathbf{2 b}$ to give sulfonium ions (9a-c). Treatment of compounds 9a-c with $\mathrm{Et}_{2} \mathrm{NH}$ or $\mathrm{Et}_{3} \mathrm{~N}$ gave corresponding sulfide products (10a-c).
\end{abstract}




\section{INTRODUCTION}

Dimethyl sulfide ditriflate (DMSD) ${ }^{1}$ is a good electrophile, which is useful for introduction of methylthio group in aromatic compounds. $2 H$-Cyclohepta[b]furan-2-ones, ${ }^{2}$ which have heptafulven and heteroazulene structures, ${ }^{3}$ react with electrophiles such as arylaldehydes ${ }^{4}$ and 1-trifuruoromethanesulfonylpyridinium trifluoromethanesulfonate ${ }^{5}$ to afford triarylmethanes and dihydropyridinyl-substituted products, respectively. During the investigation of electrophilic substitution using $\mathrm{Tf}_{2} \mathrm{O}$ in $2 \mathrm{H}$-cyclohepta[b]furan-2-ones, we found new coupling reactions of $2 H$-cyclohepta[b]furan-2-one rings. ${ }^{6}$

\section{RESULTS AND DISCUSSION}

Methyl 2-oxo-2H-cyclohepta[b]furan-3-carboxylates $(\mathbf{1 a}, \mathbf{b})$ were selected as starting materials. Methoxycarbonyl group was easily removed with $85 \% \mathrm{H}_{2} \mathrm{SO}_{4}$ to afford $2 \mathrm{H}$-cyclohepta[b]furan-2-ones $(\mathbf{2 a}, \mathbf{b}){ }^{2}$ 2a could be recrystallized from EtOH, but $\mathbf{2 b}$ was oil at room temperature. 2a and $\mathbf{2 b}$ reacted with DMSD to afford aryl dimethyl sulfoniumu trifluoromethanesulfonate derivatives (3a,b) as greenish crystals. Although 3a was recrystallized from $\mathrm{MeOH}$, 3b couldn’t because of its high solubility. These sulfonium compounds (3a,b) dissolved in ${ }^{i} \mathrm{PrOH}$ were treated with $\mathrm{Et}_{2} \mathrm{NH}$ or $\mathrm{Et}_{3} \mathrm{~N}$ at $100{ }^{\circ} \mathrm{C}$ to give $4 \mathbf{a}$ and $\mathbf{4 b}$, respectively. ${ }^{7}$ These sulfides $\mathbf{( 4 a , b )}$ were easily oxidized with $m$-CPBA to give 3-methylsulfinyl-2H-cyclohepta[b]furan-2-ones $(\mathbf{5 a} \mathbf{a}, \mathbf{b})$ as yellow crystals. Furthermore $\mathbf{5 a}$ and $\mathbf{5 b}$ were oxidized with anther $m$-CPBA to afford $\mathbf{6 a}$ and $\mathbf{6 b}$ within $2 \mathrm{~h}$, respectively.

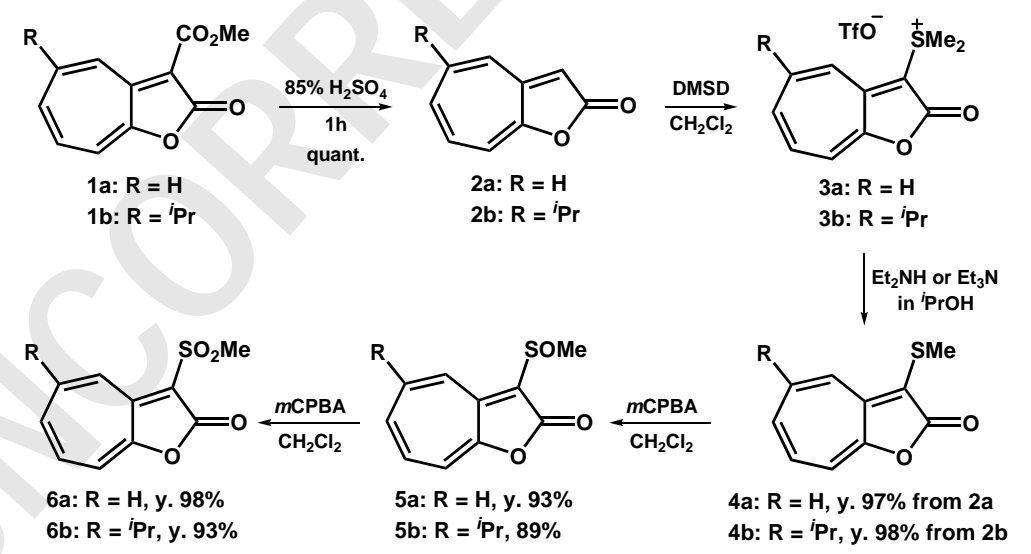

Scheme 1

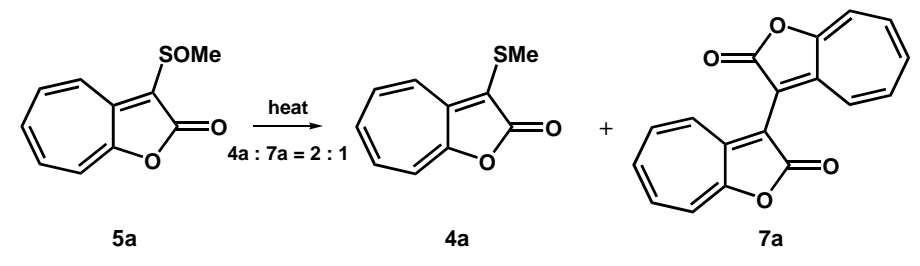

Scheme 2 


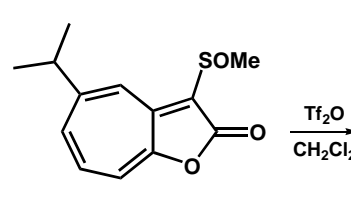

$5 b$

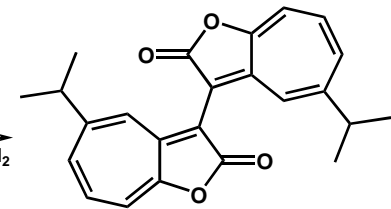

7b: y. $68 \%$

\section{Scheme 3}

We thought that diaryl sulfides (10a-c) can be obtained from sulfoxides (5a,b) similarly. Sulfoxide $\mathbf{5 b}$ dissolved in $\mathrm{CH}_{2} \mathrm{Cl}_{2}$ was reacted with $\mathrm{Tf}_{2} \mathrm{O}$. When $\mathrm{Tf}_{2} \mathrm{O}$ was dropped into the solution, the solution turned from yellow to dark brown. After the purification, unexpected product (7b) was obtained as brown plates (Scheme 3). The structure of $\mathbf{7 b}$ was determined by spectral data. Similarly $\mathbf{5 a}$ was treated with $\mathrm{Tf}_{2} \mathrm{O}$. However, only unidentified products were obtained. Product 7a was prepared by heating 5a at $40{ }^{\circ} \mathrm{C}$ under reduced pressure (Scheme 2), along with 4a (4a: 7a = $2: 1)$. Although the mechanism is not clear, we supposed that the reaction was taken place by heat and/or in the presence of small amount of acid. There are few reports concerning aryl-aryl coupling of aromatic compounds with sulfur as far as we know. Although it is necessary to optimize their condition, this reaction will be applicable for preparing electron rich bi-aryl compounds.

Sulfoxide 5a, sulfonium $\mathbf{8 a}$ and $\mathbf{8 b}$ might be unstable at even room temperature, therefore the reaction was carried out at $-80{ }^{\circ} \mathrm{C}$ in the presence of $\mathbf{2} \mathbf{b}$. As diaryl methyl sulfonium ion $\mathbf{9 b}$ was considered stable compound compared with $\mathbf{8 a}$ and $\mathbf{8 b}, \mathbf{2 b}$ was reacted immediately with aryl methyl sulfoniumu ditrifulate $\mathbf{8 b}$ to give $\mathbf{9 b}$ (Scheme 4). The reaction mixture was treated with $\mathrm{Et}_{3} \mathrm{~N}$ to afford bis(5-isopropyl-2-oxo-2H-cyclohepta[b]furan-3-yl) sulfide (10b) as orange needles. In addition, the other sulfides (10a,c) were prepared via diaryl methyl sulfonium cation (9a,c) under the same condition. Although 10b and 10c dissolve in various solvents due to isopropyl group, 10a dose not.

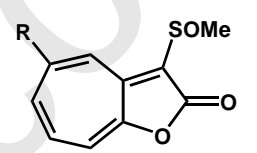

5a: $R=H$,

5b: $\mathbf{R}={ }^{i} \mathrm{Pr}$

1) $2 a$ or $2 b$

2) $\mathrm{Tf}_{2} \mathrm{O}$ at $-80^{\circ} \mathrm{C}$

$\stackrel{\mathrm{Tf}_{2} \mathrm{O} \text { at }-80^{\circ} \mathrm{C}}{\longrightarrow}$
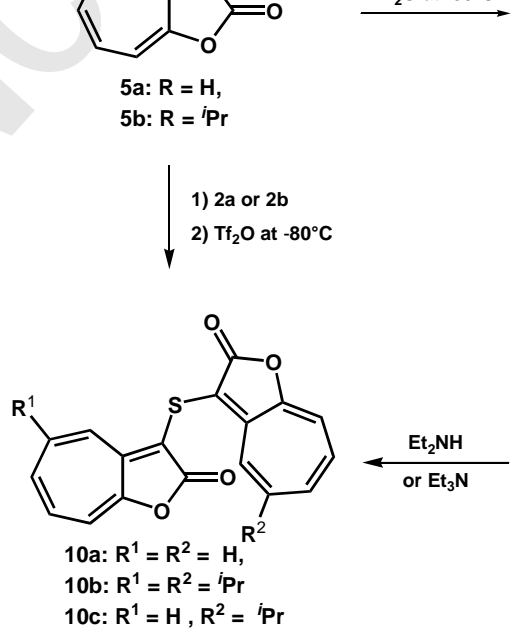

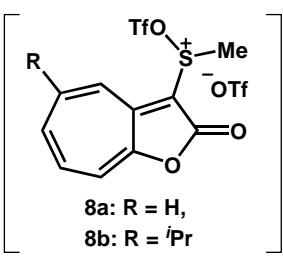

$\checkmark$ 2a or 2b

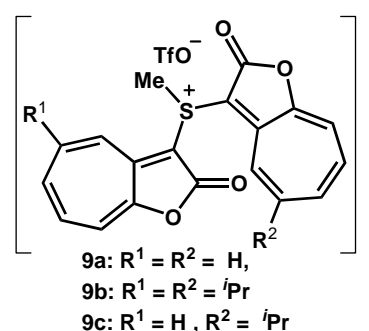

Scheme 4 


\section{Spectral properties of $2 \mathrm{H}$-cyclohepta[b]furan-2-ones}

The chemical shifts of the ring protons in $2 \mathrm{H}$-cyclohepta[b]furan-2-ones are determined on the basis of H-H COSY as shown in Table1.

Table 1. Chemical Shifts of ring proton (ppm)

\begin{tabular}{c|ccccc}
\hline \multirow{2}{*}{ Compound } & \multicolumn{6}{|c}{ Position } \\
& 4 & 5 & 7 & 8 & 6 \\
\hline 2a & 7.32 & 7.05 & 7.03 & 6.97 & 6.84 \\
3a* & 8.09 & 8.01 & 7.89 & 7.86 & 7.72 \\
$4 \mathrm{a}$ & 7.51 & 7.13 & 7.02 & 6.95 & 6.86 \\
$5 \mathrm{a}$ & 8.35 & 7.45 & 7.36 & 7.35 & 7.22 \\
$6 \mathrm{a}$ & 8.73 & 7.69 & 7.58 & 7.62 & 7.44 \\
7a & 7.45 & 7.17 & 7.12 & 7.11 & 6.96 \\
$10 \mathrm{a}$ & 8.21 & 7.36 & 7.13 & 7.08 & 7.01 \\
\hline *: Messured in $\mathrm{CD}_{3} \mathrm{CN}$ & & &
\end{tabular}

\begin{tabular}{c|cccc}
\hline \multirow{2}{*}{ Compound } & \multicolumn{4}{|c}{ Position } \\
\cline { 2 - 5 } & 4 & 7 & 8 & 6 \\
\hline 2b & 7.21 & 6.97 & 6.87 & 6.77 \\
3b* & 8.13 & 7.71 & 7.66 & 7.57 \\
4b & 7.44 & 7.00 & 6.88 & 6.81 \\
5b & 8.20 & 7.32 & 7.27 & 7.16 \\
6b & 8.70 & 7.55 & 7.52 & 7.40 \\
7b & 7.31 & 7.05 & 7.01 & 6.87 \\
10b & 8.20 & 7.07 & 6.97 & 6.94 \\
\hline
\end{tabular}

Table 2. $\Delta \delta$ values of ring proton: $\Delta \delta=\delta$ (products) $-\delta$ (2a,b)

\begin{tabular}{c|rrrrr}
\hline \multirow{2}{*}{ Compound } & \multicolumn{6}{|c}{ Position } \\
& \multicolumn{1}{|c}{4} & \multicolumn{1}{c}{7} & \multicolumn{1}{c}{8} & \multicolumn{1}{c}{6} \\
\hline 3a* & 0.77 & 0.96 & 0.86 & 0.89 & 0.88 \\
$4 \mathrm{a}$ & 0.19 & 0.08 & -0.01 & -0.02 & 0.02 \\
$5 \mathrm{a}$ & 1.03 & 0.40 & 0.33 & 0.38 & 0.38 \\
$6 \mathrm{a}$ & 1.41 & 0.64 & 0.55 & 0.65 & 0.60 \\
$7 \mathrm{a}$ & 0.13 & 0.12 & 0.09 & 0.14 & 0.12 \\
$10 \mathrm{a}$ & 0.89 & 0.31 & 0.10 & 0.11 & 0.17 \\
\hline
\end{tabular}

\begin{tabular}{c|cccc}
\hline \multirow{2}{*}{ Compound } & \multicolumn{4}{|c}{ Position } \\
& 4 & 7 & 8 & 6 \\
\hline $3 b^{*}$ & 0.92 & 0.74 & 0.79 & 0.80 \\
$4 \mathrm{~b}$ & 0.23 & 0.03 & 0.01 & 0.04 \\
$5 \mathrm{~b}$ & 0.99 & 0.35 & 0.40 & 0.39 \\
6b & 1.49 & 0.58 & 0.65 & 0.63 \\
7b & 0.10 & 0.08 & 0.14 & 0.10 \\
$10 \mathrm{~b}$ & 0.99 & 0.10 & 0.10 & 0.17 \\
\hline
\end{tabular}

* : Messured in $\mathrm{CD}_{3} \mathrm{CN}$

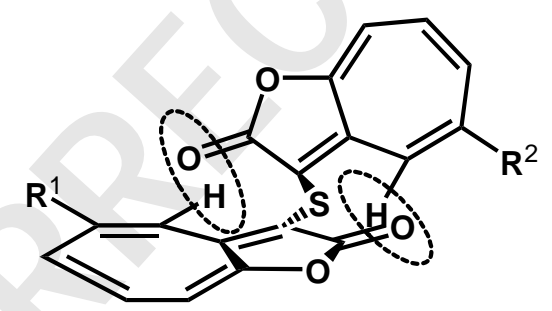

\section{Chart 1}

The $\Delta \delta$ values of products are shown Table 2. Dimethylsulfonium, methylsulfinyl and methylsulfonyl groups behave as electron-withdrawing group (EWG). In sulfonium cations (3a,b), the chemical shifts of all ring protons shifted to downfield by 1.0-0.7 ppm compared with $\mathbf{2 a}$ and $\mathbf{2 b}$. In sulfoxides (5a,b) and sulfones (6a,b), the chemical shifts at the 4-position only shifted to downfield more by 0.86-0.63 ppm. It was caused by the anithotropy of sulfinyl or sulfonyl group. The $\Delta \delta$ values of sulfides $(\mathbf{4 a}, \mathbf{b})$ are very small, but the shift at the 4-position is larger than any other position. It is caused by the interaction (ex. hydrogen bond) of lone pair electrons on sulfur. Similar tendency was observed in 3,3'-bi(2H-cyclohepta[b]furan-2-one-3-yl)s (7a,b). In $\mathbf{7 a}$ and $\mathbf{7 b}$, the conjugation caused downfield shift. However diaryl sulfides (10a-c) showed suspicious behavior. The chemical shifts at the 4 and 5-position extraordinarily shifted to downfield. Such phenomena are caused by the anisotropy of its carbonyl group through space (Chart 1). 

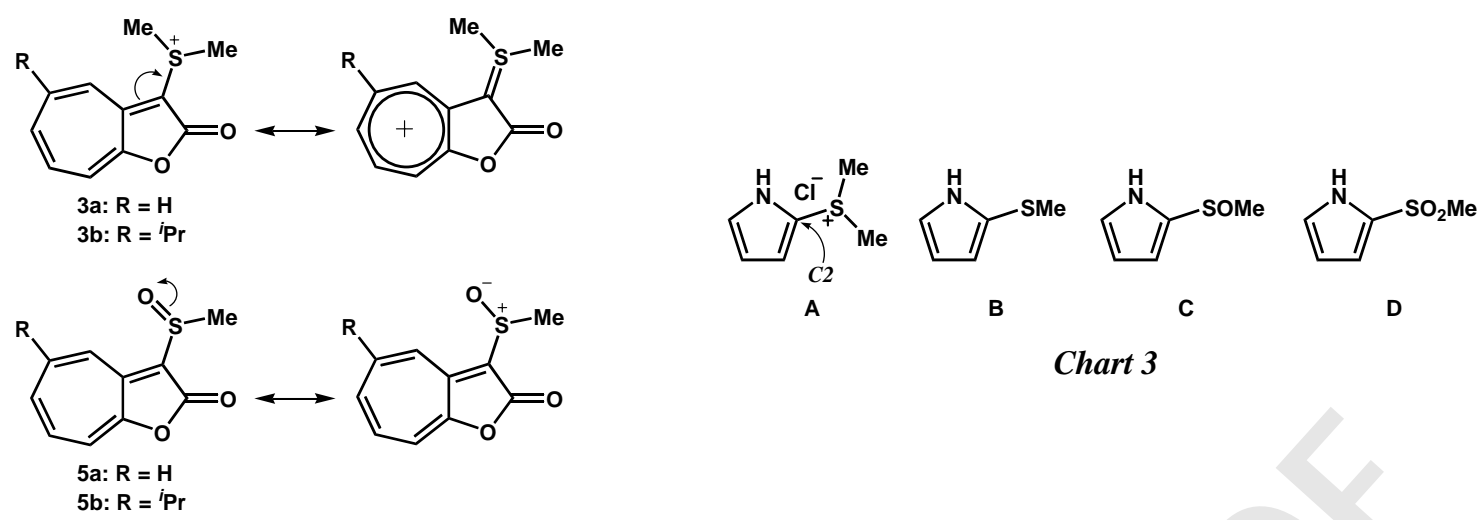

Chart 3

Chart 2

Table 3. ${ }^{13} \mathrm{C}$ NMR Chemical Shifts in $\mathrm{CD}_{3} \mathrm{Cl}(\mathrm{ppm})$

\begin{tabular}{c:ccccccccccccccc}
\hline Position & $\mathbf{A}$ & $\mathbf{B}$ & $\mathbf{C}$ & $\mathbf{D}$ & & Position & 3a* & $\mathbf{4 a}$ & $\mathbf{5 a}$ & $\mathbf{6 a}$ & Position & 3b & $\mathbf{4 b}$ & $\mathbf{5 b}$ & $\mathbf{6 b}$ \\
\hline $\mathrm{C} 2$ & 103.6 & 121.3 & 128.1 & 127.1 & & $\mathrm{C} 3$ & 82.77 & 104.72 & 107.02 & 104.25 & $\mathrm{C} 3$ & - & 103.29 & 105.38 & 102.45 \\
$\mathrm{CH}_{3}$ & 31.0 & 21.9 & 49.0 & 45.6 & $\mathrm{CH}_{3}$ & 26.62 & 16.17 & 38.43 & 43.20 & & $\mathrm{CH}_{3}$ & - & 16.30 & 37.83 & 43.17 \\
\hline
\end{tabular}

* : Messured in $\mathrm{CD}_{3} \mathrm{CN}$

In also ${ }^{13} \mathrm{C}$ NMR spectra, interesting phenomena were observed (Table 3). In general, the chemical shift of carbon substituted with EWG appears in downfield. Interestingly however, the chemical shifts at 3-pisition increased in following order: (ppm); 3a (82.77)<6a $(104.25)<\mathbf{4 a}(104.72)<5 \mathbf{a}(107.02)$, and 6b $(102.45)<\mathbf{4 b}(103.29)<5 \mathbf{b}(105.38)$. It seems that dimethylsulfonium group is electron-donating group, and sulfinyl group is EWG. This observation is explained as follows. The 3-carbon in 3a would be sealed magnetically to cause upper field shift because of the contribution of ylene form (Chart 2). Sulfinyl groups can polarize, and then those groups inductively withdrew electrons to deshield the carbons. The similar phenomena were observed in pyrrole derivertives (A-D, shown in Chart 3). ${ }^{8}$ The chemical shifts at the 2-pisition are 103.6, 121.3, 128.1 and $127.1 \mathrm{ppm}$, respectively in this order (Table 3). In contrast, the chemical shifts of methyl group in our compounds increased in following order: (ppm); 4a (16.17)<3a $(26.62)<5 \mathbf{a}(38.42)<\mathbf{6 a}(43.20)$ and $\mathbf{4 b}(16.30)<5 \mathbf{b}(37.83)<\mathbf{6 b}$ (43.17). In the pyrrole derivatives, the chemical shifts of methyl group are 31.0, 21.9, 49.0 and $45.6 \mathrm{ppm}$, respectively. These data suggest that both sulfinyl and sulfonyl group are EWG. Such a significant difference in NMR spectra might be caused by the difference of alkyl and aryl group.

2H-Cyclohepta[b]furan-2-ones (2a and $\mathbf{2 b}$ ) have tow absorptions at 374 and $389 \mathrm{~nm}$, respectively (Table 4). Introduction of methylthio, methylsulfinyl and methylsulfonyl groups caused redshift slightly. Absorption maximum of diaryl sulfides (10a-c) shifted from $374 \mathrm{~nm}$ to about $420 \mathrm{~nm}$ by 22-46 nm. These red-shifts might be caused by the intramolecular interaction. In 3,3'-bi(2-oxo-2H-cyclohepta[b]furan-2-one-3-yl)s (7a and $7 \mathbf{b}$ ), $\lambda_{\max }$ appeared around $450 \mathrm{~nm}$ due to an expansion of the $\pi$-conjugation. This suggests that the dihedral angels in $\mathbf{7 a}$ or $\mathbf{7 b}$ are small. 


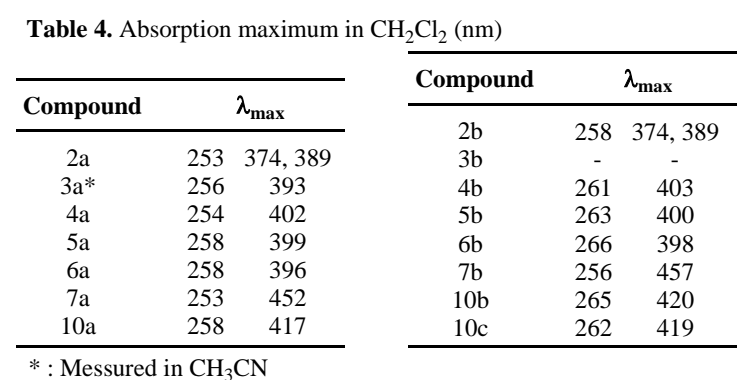

\section{EXPERIMENTAL}

General: Melting points were determined with a Yanagimoto MPS3 micro melting apparatus and are uncorrected. Mass spectra were obtained with a JEOL HX-110, a Hitachi M-2500, or a Bruker APEX II instrument, usually at $70 \mathrm{eV}$. IR and UV spectra were measured with a Shimadzu FTIR-8100M and a Hitachi U-3410 spectrophotometer, respectively. ${ }^{1} \mathrm{H}$ and ${ }^{13} \mathrm{C}$ NMR spectra were recorded with a JEOL GSX 400 (400 and $100 \mathrm{MHz}$ ), or a Bruker AM 600 spectrometer (600 and $150 \mathrm{MHz}$ ). Elemental analyses were performed at the Research and Analytical Center for Giant Molecules, Graduate School of Science, Tohoku University.

Synthesis of (2-oxo-2H-cyclohepta[b]furan-3-yl)dimethylsulfonium trifluoromethanesulfonate (3a): The solution of $\mathrm{Tf}_{2} \mathrm{O}(2.13 \mathrm{~g}, 7.50 \mathrm{mmol})$ dissolved in $\mathrm{CH}_{2} \mathrm{Cl}_{2}(20 \mathrm{~mL})$ was added dropwise to a solution of 2a (742 mg, $5.08 \mathrm{mmol})$ dissolved in $\mathrm{CH}_{2} \mathrm{Cl}_{2}(40 \mathrm{~mL})$. The solvent was removed under reduced pressure. The residue was purified by recrystallization from $\mathrm{MeOH}$. 3a (1.27 g, 3.56 mmol, 70 \%) was obtained as green needle crystals.

mp 150-152 ${ }^{\circ} \mathrm{C}$; HRMS (ESI): Calcd for $\mathrm{C}_{11} \mathrm{H}_{11} \mathrm{O}_{2} \mathrm{~S}^{+}[\mathrm{M}]^{+}$207.0474. Found: 207.0473; IR (KBr disk): $v_{\max } 3042(\mathrm{~m}), 3001(\mathrm{w}), 2970(\mathrm{w}), 2943$ (w), $2889(\mathrm{w}), 1859(\mathrm{w}), 1782$ (m), 1742 (s, C=O), 1692 (m), 1616 (w), 1597 (m), 1541 (m), 1481 (s), 1466 (s), 1431 (s), 1404 (m), 1327 (m), 1281 (s), 1258 (s), 1223 (s), 1159 (s), 1065 (m), 1053 (m), 1028 (s), 997 (s), 955 (m), 937 (m), 891 (w), 868 (w), 768 (s), 718 (m), 629 (s), 573 (m), 517 (m), $486(\mathrm{w}), 426(\mathrm{w}), 411$ (w) cm ${ }^{-1}$; UV/Vis $\left(\mathrm{CH}_{3} \mathrm{CN}\right): \lambda_{\max }, \mathrm{nm}(\log \varepsilon) 219$ (4.31), 256 (4.41), 260 sh (4.39), 393 (4.35); ${ }^{1} \mathrm{H}$ NMR (600 MHz, CD ${ }_{3} \mathrm{CN}$ ): $\delta 8.09$ (d, $\left.J=11.0 \mathrm{~Hz}, 1 \mathrm{H}, \mathrm{H}-4\right)$, 8.01 (ddd, $J=11.0,9.5,2.0$,1H, H-5), 7.89 (dd, $J=9.5,2.0$ Hz, 1H, H-8), 7.86 (t, $J=9.5$ Hz, 1H, H-7), 7.72(ddt, $J=9.5,2.0,0.7 \mathrm{~Hz}, 1 \mathrm{H}, \mathrm{H}-6), 3.24$ (s, $\left.6 \mathrm{H},-\mathrm{S}^{+}\left(\mathrm{C}_{3}\right)_{2}\right) ;{ }^{13} \mathrm{C}$ NMR $\left(150 \mathrm{MHz}, \mathrm{CD}_{3} \mathrm{CN}\right): \delta 164.61$ (C-2), 158.67 (C-8a or 3a), 156.08 (C-8a or 3a), 142.66 (C-5), 139.20 (C-8), 137.68 (C-6), 130.61 (C-4), 123.01 (C-7), 121.95 (q, $\left.J=318 \mathrm{~Hz},-\mathrm{CF}_{3}\right), 82.77$ (C-3), $26.62\left(-\mathrm{CH}_{3}\right)$; Anal. Calcd for $\mathrm{C}_{12} \mathrm{H}_{11} \mathrm{~F}_{3} \mathrm{O}_{5} \mathrm{~S}_{2}$ : C, 40.45; H, 3.11. Found: C, 40.386; H, 3.214.

Synthesis of (5-isopropyl-2oxo-2H-cyclohepta[b]furan-3-yl)dimethylsulfonium trifluoromethanesulfonate (3b): The solution of $\mathrm{Tf}_{2} \mathrm{O}(883 \mathrm{mg}, 3.11 \mathrm{mmol})$ dissolved in $\mathrm{CH}_{2} \mathrm{Cl}_{2}(10 \mathrm{~mL})$ was added dropwise to a solution of $\mathbf{2 b}(391 \mathrm{mg}, 2.08 \mathrm{mmol})$ dissolved in $\mathrm{CH}_{2} \mathrm{Cl}_{2}(10 \mathrm{~mL})$. The solvent was removed under reduced pressure. $\mathbf{3 b}$ was obtained as green needle crystals.

${ }^{1} \mathrm{H}$ NMR (400 MHz, $\mathrm{CDCl}_{3}$ ): $\delta 8.13$ (d, $\left.J=1.2 \mathrm{~Hz}, 1 \mathrm{H}, \mathrm{H}-4\right), 7.71$ (d, $J=9.6,9.6 \mathrm{~Hz}, 1 \mathrm{H}, \mathrm{H}-7$ ), 7.66 (dd, 
$J=9.6,1.2 \mathrm{~Hz}, 1 \mathrm{H}, \mathrm{H}-8), 7.57$ (ddd, $J=9.6,1.2,1.2 \mathrm{~Hz}, 1 \mathrm{H}, \mathrm{H}-6), 3.35$ (s, $\left.6 \mathrm{H},-\mathrm{S}^{+}\left(\mathrm{CH}_{3}\right)_{2}\right), 3.25$ (sept, $J$ $\left.=6.8,1 \mathrm{H},-\mathrm{C} \underline{\mathrm{H}}\left(\mathrm{CH}_{3}\right)_{2}\right), 1.39\left(\mathrm{~d}, J=6.8 \mathrm{~Hz}, 6 \mathrm{H},-\mathrm{CH}\left(\mathrm{CH}_{3}\right)_{2}\right)$

Synthesis of 3-methylthio-2H-cyclohepta[b]furan-2-one (4a): The solution of $\mathrm{Tf}_{2} \mathrm{O}$ (4.39 $\mathrm{g}, 15.5$ mmol) dissolved in $\mathrm{CH}_{2} \mathrm{Cl}_{2}(40 \mathrm{~mL})$ was added dropwise to a solution of 2a (1.50 g, $\left.10.3 \mathrm{mmol}\right)$ and DMSO (1.61 g, $20.6 \mathrm{mmol})$ dissolved in $\mathrm{CH}_{2} \mathrm{Cl}_{2}(40 \mathrm{~mL})$ at $0{ }^{\circ} \mathrm{C}$ follwed by stirring for $10 \mathrm{~min}$. $\mathrm{Et}_{3} \mathrm{~N}(40$ $\mathrm{mL}$ ) was added to the solution and refluxed at $70{ }^{\circ} \mathrm{C}$ for $20 \mathrm{~min}$. The solvent was removed under reduced pressure. The residue was extracted with $\mathrm{CH}_{2} \mathrm{Cl}_{2} / \mathrm{H}_{2} \mathrm{O}$, dried over $\mathrm{MgSO}_{4}$ and purified on silica gel column chromatography with $\mathrm{CH}_{2} \mathrm{Cl}_{2}$. $4 \mathbf{a}(1.91 \mathrm{~g}$, $9.95 \mathrm{mmol}, 97$ \%) was obtained as red crystals.

mp 68-69 ${ }^{\circ} \mathrm{C}$; HRMS (ESI): Calcd for $\mathrm{C}_{10} \mathrm{H}_{8} \mathrm{O}_{2} \mathrm{~S}+\mathrm{Na}[\mathrm{M}+\mathrm{Na}]^{+}$215.0137. Found: 215.0137; IR ( $\mathrm{KBr}$ disk): v $v_{\max } 3069(\mathrm{w}), 3049(\mathrm{w}), 3038(\mathrm{w}), 3018(\mathrm{w}), 2997(\mathrm{w}), 2922(\mathrm{~m}), 2862(\mathrm{w}), 2818(\mathrm{w}), 1779(\mathrm{w})$, 2764 (w), 1767 (m), 1728 (s, C=O), 1678 (m), 1595 (s), 1528 (s), 1504 (s), 1464 (m), 1412 (m), 1308 (m), 1294 (m), 1265 (s), 1232 (s), 1148 (m), 1049 (m), 1003 (w), 989 (w), 978 (m), 959 (m), 947 (m), 891 (m), 864 (m), 843 (w), 758 (s), 737 (m), 702 (m), 629 (m), 611 (w), 581 (w), 486 (w), 449 (w) cm ; UV/Vis $\left(\mathrm{CH}_{2} \mathrm{Cl}_{2}\right): \lambda_{\max }, \mathrm{nm}(\log \varepsilon) 254$ (4.26), 402 (4.20); ${ }^{1} \mathrm{H}$ NMR (600 MHz, $\left.\mathrm{CDCl}_{3}\right): \delta 7.51$ (bd, $1 \mathrm{H}, J=11.2$ Hz, H-4), 7.13 (ddd, 1H, $J=11.2,8.5,0.8$ Hz, H-5), 7.02 (ddd, 1H, $J=11.0,9.0,0.8$ Hz, H-7), 6.95 (dd, $J=9.0,0.9 \mathrm{~Hz}, 1 \mathrm{H}, \mathrm{H}-8), 6.86$ (dddd, $1 \mathrm{H}, J=11.0,8.5,0.9,0.7 \mathrm{~Hz}, \mathrm{H}-6), 2.42$ (s, 3H, -SMe); ${ }^{13} \mathrm{C}$ NMR (100 MHz, $\mathrm{CDCl}_{3}$ ): $\delta 167.50$ (C-2), 157.45 (C-8a), 151.27 (C-3a), 135.29 (C-5), 132.74 (C-7), 130.89 (C-6), 128.02 (C-4), 113.68 (C-8), 104.72 (C-3), 16.17 (-SMe); Anal. Calcd for $\mathrm{C}_{10} \mathrm{H}_{8} \mathrm{O}_{2} \mathrm{~S}$ : C, 62.48; H, 4.19. Found: C, 62.661; H, 4.321.

Synthesis of 5-isopropyl-3-methylthio-2H-cyclohepta[b]furan-2-one (4b): The solution of $\mathrm{Tf}_{2} \mathrm{O}$ (4.25 g, $15.0 \mathrm{mmol})$ dissolved in $\mathrm{CH}_{2} \mathrm{Cl}_{2}(25 \mathrm{~mL})$ was added dropwise to a solution of $\mathbf{2 b}(1.53 \mathrm{~g}, 8.13 \mathrm{mmol})$ and DMSO (1.17 g, $15.0 \mathrm{mmol})$ dissolved in $\mathrm{CH}_{2} \mathrm{Cl}_{2}(40 \mathrm{~mL})$. The solvent was removed under reduced pressure. $\mathrm{CH}_{2} \mathrm{Cl}_{2}(30 \mathrm{~mL})$ and $\mathrm{Et}_{2} \mathrm{NH}(15 \mathrm{~mL})$ was added to the residue followed by stirring for a few minutes. The solvent was removed under reduced pressure. The residue was purified on silica gel chromatography with $\mathrm{CH}_{2} \mathrm{Cl}_{2}$. 4b (1.87 g, $7.97 \mathrm{mmol}$, $98 \%$ ) was obtained as orange oil.

HRMS (ESI): Calcd for $\mathrm{C}_{13} \mathrm{H}_{14} \mathrm{O}_{2} \mathrm{~S}+\mathrm{Na}[\mathrm{M}+\mathrm{Na}]^{+}$257.0607. Found: 257.0607; IR (KBr disk): $v_{\max }$ 3034 (w), 2961 (m), 2926 (m), 2872 (m), 1987 (w), 1858 (w), 1736 (s), 1628 (w), 1595 (s), 1507 (s), 1495 (s), 1420 (s), 1381 (w), 1364 (w), 1296 (m), 1271 (s), 1237 (s), 1129 (w), 1073 (w), 1053 (m), 1040 (m), $1019(\mathrm{w}), 968$ (w), 947 (w), $928(\mathrm{w}), 909(\mathrm{~m}), 862(\mathrm{w}), 799(\mathrm{~m}), 756(\mathrm{~m}), 718(\mathrm{w}), 696(\mathrm{w}), 652(\mathrm{w})$, 627 (w), 455 (w) cm ${ }^{-1}$; UV/Vis $\left(\mathrm{CH}_{2} \mathrm{Cl}_{2}\right): \lambda_{\max }, \mathrm{nm}$ (loge) 240 sh (4.22), 261 (4.33), 403 (4.23); ${ }^{1} \mathrm{H}$ NMR (600 MHz, $\mathrm{CDCl}_{3}$ ): $\delta 7.44$ (dd, $\left.1 \mathrm{H}, J=1.4,0.7 \mathrm{~Hz}, \mathrm{H}-4\right), 7.00$ (ddd, 1H, $J=11.5,9.0,0.5 \mathrm{~Hz}, \mathrm{H}-7$ ), 6.88 (dd, 1H, $J=9.0,0.9 \mathrm{~Hz}, \mathrm{H}-8$ ), 6.81 (ddd, 1H, $J=11.5,1.4,0.7 \mathrm{~Hz}, \mathrm{H}-6$ ), 2.89 (sept, 1H, $J=6.8$,

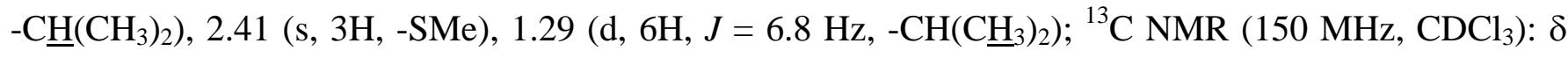
167.78 (C-2), 157.10 (C-5), 156.77 (C-8a), 151.21 (C-3a), 132.17 (C-7 or 6), 132.04 (C-7 or 6), 124.66 
(C-4), 112.78 (C-8), 103.29 (C-3), 38.97 (- $\left.\underline{\mathrm{CH}}\left(\mathrm{CH}_{3}\right)_{2}\right), 23.05$ (-CH( $\left.\left.\underline{\mathrm{C}} \mathrm{H}_{3}\right)_{2}\right), 16.30$ (-SMe); Anal. Calcd for $\mathrm{C}_{13} \mathrm{H}_{14} \mathrm{O}_{2} \mathrm{~S}$ : C, 66.64; H, 6.02. Found: C, 66.644; H, 6.033.

Synthesis of 3-methylsulfinyl-2H-cyclohepta[b]furan-2-one (5a): m-CPBA (212 mg, $1.23 \mathrm{mmol}$ ) was added to the solution of $\mathbf{4 a}$ (214 $\mathrm{mg}, 1.11 \mathrm{mmol})$ dissolved in $\mathrm{CHCl}_{3}(10 \mathrm{~mL})$ followed by stirring for a few minutes. The solution was extracted with $10 \% \mathrm{~K}_{2} \mathrm{CO}_{3}$ solution, dried over $\mathrm{MgSO}_{4}$ and evaporated under reduced pressure. 5a (214 mg, $1.03 \mathrm{mmol}$, 93 \%) was obtained as yellow crystals.

mp 117-119 ${ }^{\circ} \mathrm{C}$; HRMS (ESI): Calcd for $\mathrm{C}_{10} \mathrm{H}_{8} \mathrm{O}_{3} \mathrm{~S}+\mathrm{Na}[\mathrm{M}+\mathrm{Na}]^{+}$231.086. Found: 231.0085; IR ( $\mathrm{KBr}$ disk): $v_{\max } 3056(\mathrm{w}), 3029$ (w), 3015 (w), 3004 (w), 2923 (w), 1775 (m), 1740 (s), 1532 (m), 1493 (m), 1483 (m), 1462 (m), 1422 (m), 1404 (m), 1294 (w), 1271 (m), 1242 (m), 1229 (m), 1146 (w), 1057 (w), 1028 (s), 953 (m), 943 (m), 887 (w), 860 (w), 768 (m), 749 (m), 716 (m), 687 (w), $629(\mathrm{~m}), 612(\mathrm{w}), 482$ (w), 459 (w), $430(\mathrm{w}), 419(\mathrm{w}), 403(\mathrm{w}) \mathrm{cm}^{-1}$; UV/Vis $\left(\mathrm{CH}_{2} \mathrm{Cl}_{2}\right): \lambda_{\max }, \mathrm{nm}(\log \varepsilon) 258$ (4.34), 399 (4.26); ${ }^{1} \mathrm{H}$ NMR (600 MHz, $\mathrm{CDCl}_{3}$ ): $\delta 8.35$ (bd, $\left.1 \mathrm{H}, J=11.2 \mathrm{~Hz}, \mathrm{H}-4\right), 7.45$ (ddd, $1 \mathrm{H}, J=11.2,8.8,0.5 \mathrm{~Hz}, \mathrm{H}-5$ ), 7.36 (dd, 1H, $J=11.2,9.2$ Hz, H-7), 7.35 (bd, 1H,J = 9.2 Hz, H-8), 7.22 (dddd, 1H, $J=11.2$, 8.8, 1.3, 0.9, H-6), 3.14 (s, 3H, -SOMe); ${ }^{13} \mathrm{C}$ NMR (150 MHz, $\mathrm{CDCl}_{3}$ ): $\delta 163.53$ (C-2), 157.41 (C-8a), 152.59 (C-3a), 138.15 (C-5), 134.84 (C-7), 133.39 (C-6), 127.42 (C-4), 118.20 (C-8), 107.02 (C-3), 38.43 (-SOMe); Anal. Calcd for $\mathrm{C}_{10} \mathrm{H}_{8} \mathrm{O}_{3} \mathrm{~S}$ : C, 57.68; H, 3.87. Found: C, 57.290; H, 4.047.

Synthesis of 5-isopropyl-3-methylsulfinyl-2H-cyclohepta[b]furan-2-one (5b): $m$-CPBA (95.7 mg, $0.553 \mathrm{mmol})$ was added to the solution of $\mathbf{4 b}(118 \mathrm{mg}, 0.504 \mathrm{mmol})$ dissolved in $\mathrm{CH}_{2} \mathrm{Cl}_{2}(8 \mathrm{~mL})$ followed by stirring for a few minutes. The solution was extracted with $10 \% \mathrm{NaOH}$ solution, dried over $\mathrm{K}_{2} \mathrm{CO}_{3}$ and evaporated under reduced pressure. The residue was purified on silica gel column chromatography with EtOAc. $5 \mathbf{b}$ (108 mg, $0.449 \mathrm{mmol}, 89$ \%) was obtained as yellow crystals.

m.p. 88.5-90 ${ }^{\circ} \mathrm{C}$; HRMS (ESI): Calcd for $\mathrm{C}_{13} \mathrm{H}_{14} \mathrm{O}_{3} \mathrm{~S}+\mathrm{Na}[\mathrm{M}+\mathrm{Na}]^{+}$273.0556. Found: 273.0554; IR (KBr disk): v $v_{\max } 3011$ (w), 2965 (w), 2915 (w), 2870 (w), 1869 (w), 1794 (w), 1736 (s, C=O), 1628 (m), 1593 (m), 1512 (m), 1489 (m), 1466 (m), 1458 (m), 1429 (w), 1414 (w), 1381 (w), 1364 (w), $1321(\mathrm{w})$, 1294 (w), 1277 (m), 1192 (w), 1167 (w), 1103 (w), 1063 (w), 1044 (w), 1019 (w), 1005 (w), 999 (w), 968 (w), $947(\mathrm{w}), 901(\mathrm{w}), 864(\mathrm{w}), 806(\mathrm{w}), 754(\mathrm{w}), 735(\mathrm{w}), 722(\mathrm{w}), 695(\mathrm{w}), 650(\mathrm{w}), 627(\mathrm{~m}), 515(\mathrm{w})$, $463(\mathrm{w}), 448(\mathrm{w}), 419(\mathrm{w}), 407(\mathrm{w}) \mathrm{cm}^{-1}$; UV/Vis $\left(\mathrm{CH}_{2} \mathrm{Cl}_{2}\right): \lambda_{\max }, \mathrm{nm}(\log \varepsilon) 263$ (4.32), $400(4.20) ;{ }^{1} \mathrm{H}$ NMR (600 MHz, $\mathrm{CDCl}_{3}$ ): $\delta 8.20$ (d, 1H, $J=1.4 \mathrm{~Hz}, \mathrm{H}-4$ ), 7.32 (dd, 1H, $J=11.2,9.2 \mathrm{~Hz}, \mathrm{H}-7$ ), 7.27 (dd, $1 \mathrm{H}, J=9.2,1.1 \mathrm{~Hz}, \mathrm{H}-8$ ), 7.16 (ddd, 1H, $J=11.2,1.4,1.1 \mathrm{~Hz}, \mathrm{H}-6$ ), 3.15 (s, 3H, -SOMe), 3.00 (sept, 1H, $\left.J=6.8,-\mathrm{CH}\left(\mathrm{CH}_{3}\right)_{2}\right),, 1.31\left(\mathrm{~d}, 6 \mathrm{H}, J=6.8 \mathrm{~Hz},-\mathrm{CH}\left(\mathrm{C}_{3}\right)_{2}\right) ;{ }^{13} \mathrm{C} \mathrm{NMR}\left(150 \mathrm{MHz}, \mathrm{CDCl}_{3}\right): \delta 163.73(\mathrm{C}-2)$, 160.80 (C-5), 156.91 (C-8a), 152.49 (C-3a), 134.33 (C-7 or 6), 134.28 (C-7 or 6), 124.54 (C-4), 117.29 (C-8), 105.38 (C-3), 39.51 (- $\left.\underline{\mathrm{CH}}\left(\mathrm{CH}_{3}\right)_{2}\right)$, 37.83 (-SOMe), $23.20\left(-\mathrm{CH}\left(\underline{\mathrm{C}} \mathrm{H}_{3}\right)_{2}\right)$; Anal. Calcd for $\mathrm{C}_{13} \mathrm{H}_{14} \mathrm{O}_{3} \mathrm{~S} \square$ 0.2 $\mathrm{H}_{2} \mathrm{O}$ : C, 61.49; H, 5.72. Found: C, 61.355; H, 5.830

Synthesis of 3-methylsulfonyl-2H-cyclohepta[b]furan-2-one (6a): m-CPBA (394 mg, $2.28 \mathrm{mmol}$ ) was 
added to the solution of $\mathbf{4 a}$ (393 $\mathrm{mg}, 2.05 \mathrm{mmol}$ ) dissolved in $\mathrm{CHCl}_{3}(20 \mathrm{~mL})$. The solution was extracted with $10 \% \mathrm{~K}_{2} \mathrm{CO}_{3}$ solution, dried over $\mathrm{MgSO}_{4}$ and evaporated under reduced pressure. Another $m$-CPBA (477 mg, $2.76 \mathrm{mmol}$ ) was added to the solution of the residue dissolved in $\mathrm{CHCl}_{3}(15 \mathrm{~mL})$ followed by stirring for $1 \mathrm{~h}$. $\mathrm{Et}_{2} \mathrm{NH}(2 \mathrm{~mL})$ was added to the solution followed by evaporation. The residue was purified on silica gel column chromatography with $\mathrm{CHCl}_{3} / \mathrm{EtOAc}$ (10:1). 6a (451 mg, $2.01 \mathrm{mmol}, 98$ \%) was obtained as yellow crystals.

mp 219-221 ${ }^{\circ} \mathrm{C}$; HRMS (ESI): Calcd for $\mathrm{C}_{10} \mathrm{H}_{8} \mathrm{O}_{4} \mathrm{~S}+\mathrm{Na}[\mathrm{M}+\mathrm{Na}]^{+}$247.0036. Found: 247.0037; IR (KBr disk): $v_{\max } 3065$ (w), 3015 (w), 3006 (w), 2921 (w), 1782 (w), 1736 (s, C=O), 1686 (w), 1619 (w), 1595 (w), 1570 (m), 1536 (m), 1485 (s), 1460 (s), 1428 (w), 1406 (s), 1327 (w), 1316 (m), 1296 (s), 1266 (s), 1227 (m), 1159 (m), 1134 (m), 1123 (s), 1063 (w), 1001 (w), 982 (w), 968 (m), 943 (w), $926(\mathrm{w}), 895$ (w), $876(\mathrm{w}), 864$ (w), 797 (w), 777 (m), 754 (m), 741 (w), $714(\mathrm{w}), 625$ (m), $615(\mathrm{w}), 552(\mathrm{~s}), 521(\mathrm{w}), 475$ (w), $426(\mathrm{w}) \mathrm{cm}^{-1}$; UV/Vis $\left(\mathrm{CH}_{2} \mathrm{Cl}_{2}\right): \lambda_{\max }, \mathrm{nm}(\log \varepsilon) 258$ (4.37), $264 \mathrm{sh}$ (4.34), 396 (4.26); ${ }^{1} \mathrm{H}$ NMR (600 MHz, $\mathrm{CDCl}_{3}$ ): $\delta 8.73$ (bd, 1H, $J=11.2 \mathrm{~Hz}, \mathrm{H}-4$ ), 7.69 (ddd, 1H, J = 11.2, 9.0, $0.5 \mathrm{~Hz}, \mathrm{H}-5$ ), 7.62 (dd, 1H, $J=9.0,1.3 \mathrm{~Hz}, \mathrm{H}-8$ ), 7.58 (dddd, 1H, $J=11.2,9.0,1.1,0.5 \mathrm{~Hz}, \mathrm{H}-7$ ), 7.44 (dddd, $J=11.2,9.0,1.1,0.9$ 1H, H-6), 3.32 (s, 3H, -SO $\underline{\text { Me)}) ; ~}{ }^{13} \mathrm{C}$ NMR (150 MHz, $\mathrm{CDCl}_{3}$ ): $\delta 163.59$ (C-2), 157.47 (C-8a), 151.64 (C-3a), 1040.18 (C-5), 136.34 (C-7), 134.91 (C-6), 129.15 (C-4), 121.01 (C-8), 104.25 (C-3), 43.20 (-SO $\mathrm{SO}_{2}$ ); Anal. Calcd for $\mathrm{C}_{10} \mathrm{H}_{8} \mathrm{O}_{4} \mathrm{~S}$ : C, 53.56; H, 3.60. Found: C, 53.525; H, 3.759.

Synthesis of 5-isopropyl-3-methylsulfonyl-2H-cyclohepta[b]furan-2-one (6b): m-CPBA (213 mg, 1.23 mmol) was added to the solution of $5 \mathbf{b}(257 \mathrm{mg}, 1.03 \mathrm{mmol})$ dissolved in $\mathrm{CHCl}_{3}(10 \mathrm{~mL})$ followed by stirring for $2 \mathrm{~h}$. The solution was extracted with $10 \% \mathrm{~K}_{2} \mathrm{CO}_{3}$ solution, dried over $\mathrm{MgSO}_{4}$ and evaporated under reduced pressure. $6 \mathbf{b}$ (254 mg, $0.955 \mathrm{mmol}$, y. 93 \%) was obtained as yellow crystals.

mp 111-112 ${ }^{\circ} \mathrm{C}$; HRMS (ESI): Calcd for $\mathrm{C}_{13} \mathrm{H}_{14} \mathrm{O}_{4} \mathrm{~S}+\mathrm{Na}[\mathrm{M}+\mathrm{Na}]^{+}$289.0505. Found: 289.0504; IR (KBr disk): v $v_{\max } 3021$ (w), 3006 (w), 2977 (w), 2924 (m), 2869 (w), 1860 (w), 1744 (s, C=O), 1626 (m) 1595 (s), 1518 (s), 1509 (s), 1491 (s), 1472 (s), 1431 (m), 1426 (m), 1412 (m), 1389 (m), 1363 (m), 1331 (s), 1321 (m), 1300 (s), 1294 (s), 1271 (s), 1240 (s), 1194 (m), 1167 (m), 1132 (s), 1061 (m), 1042 (m), 970, (s), 943 (m), 936 (m), 914 (m), 874 (m), 814 (m), 801 (m), 801 (m), 777 (m), 772 (m), 760 (m), 752 (m), $720(\mathrm{w}), 660(\mathrm{w}), 623(\mathrm{w}), 596(\mathrm{~m}), 552(\mathrm{~s}), 527(\mathrm{~m}), 498(\mathrm{w}), 419(\mathrm{w}), 403(\mathrm{w}) \mathrm{cm}^{-1}$; UV/Vis $\left(\mathrm{CH}_{2} \mathrm{Cl}_{2}\right): \lambda_{\max }, \mathrm{nm}(\log \varepsilon) 266$ (4.47), 398 (4.35); ${ }^{1} \mathrm{H} \mathrm{NMR}\left(600 \mathrm{MHz}, \mathrm{CDCl}_{3}\right): \delta 8.70$ (d, $1 \mathrm{H}, J=1.4 \mathrm{~Hz}$, H-4), 7.55 (dd, 1H, $J=10.3,9.5$ Hz, H-7), 7.52 (dd, 1H, $J=9.5,2.0$ Hz, H-8), 7.40 (ddd, 1H, $J=10.3$, 2.0, $1.4 \mathrm{~Hz}, \mathrm{H}-6$ ), 3.31 (s, 3H, $-\mathrm{SO}_{2} \underline{\mathrm{CH}}_{3}$ ), 3.09 (sept, $\left.J=6.8,1 \mathrm{H},-\mathrm{C} \underline{\mathrm{H}}\left(\mathrm{CH}_{3}\right)_{2}\right), 1.35$ (d, $J=6.8 \mathrm{~Hz}, 6 \mathrm{H}$, -CH( $\left.\left.\underline{\mathrm{C}}_{3}\right)_{2}\right) ;{ }^{13} \mathrm{C}$ NMR (150 MHz, $\left.\mathrm{CDCl}_{3}\right): \delta 163.86$ (C-2), 163.43 (C-3a), 156.88 (C-8a), 151.17 (C-5), 135.88 (C-6), 135.74 (C-7), 126.55 (C-4), 119.88 (C-8), 102.45 (C-3), 43.17 (-SO $\left.{ }_{2} \mathrm{Me}\right), 39.88$

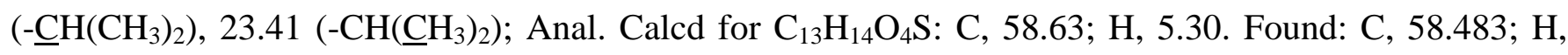
5.300 . 
Synthesis of 3,3'-bi(2-oxo-2H-cyclohepta[b]furan) (7a): 7a (16.5 mg, $0.0569 \mathrm{mmol})$ and 4a (26.9 mg, $0.140 \mathrm{mmol}$ ) were accidentally obtained by heating $5 \mathrm{a}$ at $40^{\circ} \mathrm{C}$ under reduced pressure.

Reddish brown powder; HRMS (ESI): Calcd for $\mathrm{C}_{18} \mathrm{H}_{10} \mathrm{O}_{4}+\mathrm{Na}[\mathrm{M}+\mathrm{Na}]^{+}$313.0471. Found: 313.0470; UV/Vis $\left(\mathrm{CH}_{2} \mathrm{Cl}_{2}\right): \lambda_{\max }, \mathrm{nm} 253,452 ;{ }^{1} \mathrm{H}$ NMR (400 MHz, $\left.\mathrm{CDCl}_{3}\right): \delta 7.45$ (d, 2H, $J=11.2 \mathrm{~Hz}, \mathrm{H}-4,4$ '), 7.17 (d, 2H, $J=11.2,8,8 \mathrm{~Hz}, \mathrm{H}-5,5$ ') 7.12 (dd, 2H, $J=8.8 \mathrm{~Hz}, \mathrm{H}-7,7^{\prime}$ ), 7.11 (d, 2H, $J=8.8 \mathrm{~Hz}, \mathrm{H}-8$, 8'), 6.96 (dd, 2H, $J=8.8,8.8 \mathrm{~Hz}, \mathrm{H}-6,6$ ')

Synthesis of 3,3'-bi(5-isopropyl-2H-cyclohepta[b]furan-2-one) (7b): The solution of $\mathrm{Tf}_{2} \mathrm{O}$ (174 mg, $0.612 \mathrm{mmol})$ dissolved in $\mathrm{CH}_{2} \mathrm{Cl}_{2}(10 \mathrm{~mL})$ was added dropwise to a solution of $5 \mathbf{b}$ (125 $\left.\mathrm{mg}, 0.499 \mathrm{mmol}\right)$ dissolved in $\mathrm{CH}_{2} \mathrm{Cl}_{2}(10 \mathrm{~mL})$ at $0{ }^{\circ} \mathrm{C}$. The solvent was removed under reduced pressure. The residue was extracted with $\mathrm{CH}_{2} \mathrm{Cl}_{2}$. $7 \mathbf{b}$ (63.8 mg, 0.170 mmol, 68 \%) was obtained red crystals.

mp 218-219 ${ }^{\circ} \mathrm{C}$; HRMS (ESI): Calcd for $\mathrm{C}_{24} \mathrm{H}_{22} \mathrm{O}_{4}+\mathrm{Na}[\mathrm{M}+\mathrm{Na}]^{+}$397.1410. Found: 397.1411; $\mathrm{IR}(\mathrm{KBr}$ disk): v $v_{\max } 3017$ (w), $2953(\mathrm{w}), 2930$ (w), 2903 (w), 2868 (w), 1803 (w), 1746 (s, C=O), 1722 (s), 1665 (w), 1655 (w), 1638 (w), 1597 (w), 1514 (s), 1464 (m), 1420 (w), 1389 (w), 1364 (w), 1344 (w), 1321 (w), 1281 (w), 1265 (m), 1248 (w), 1230 (m), 1202 (w), 1186 (w), 1049 (w), 1034 (w), 937 (w), $914(w), 901$ (w), $878(\mathrm{~m}), 831(\mathrm{w}), 791(\mathrm{~m}), 783(\mathrm{w}), 754(\mathrm{~m}), 710(\mathrm{w}), 644(\mathrm{w}) \mathrm{cm}^{-1}$; UV/Vis $\left(\mathrm{CH}_{2} \mathrm{Cl}_{2}\right): \lambda_{\max }, \mathrm{nm}$ (loge) 256 (4.66), 263 sh (4.64), 366 sh (3.90), 457 (4.44); ${ }^{1} \mathrm{H}$ NMR (600 MHz, $\left.\mathrm{CDCl}_{3}\right): \delta 7.31$ (d, $J=1.3$ Hz, 2H, H-4, 4’), 7.05 (dd, $J=11.0,9.2 \mathrm{~Hz}, 2 \mathrm{H}, \mathrm{H}-7,7^{\prime}$ ), 7.01 (dd, $J=9.2,1.1 \mathrm{~Hz}, 2 \mathrm{H}, \mathrm{H}-8,8^{\prime}$ ), 6.87 (ddd, $J=11.0,1.3,1.1 \mathrm{~Hz}, 2 \mathrm{H}, \mathrm{H}-6,6$ '), 2.85 (sept, $\left.J=6.8 \mathrm{~Hz}, 2 \mathrm{H},-\mathrm{C} \underline{\mathrm{H}}\left(\mathrm{CH}_{3}\right)_{2}\right), 1.26$ (d, $J=6.8 \mathrm{~Hz}, 12 \mathrm{H}$, $\left.-\mathrm{CH}\left(\underline{\mathrm{CH}}_{3}\right)_{2}\right) ;{ }^{13} \mathrm{C}$ NMR (150 MHz, $\left.\mathrm{CDCl}_{3}\right): \delta 167.19$ (C-2), 158.04, 156.52, 148.00, 132.77 (C-6), 132.26 (C-7), 126.48 (C-4), 113.99 (C-8), 99.66 (C-3), $39.07\left(-\underline{\mathrm{CH}}\left(\mathrm{CH}_{3}\right)_{2}\right), 23.2\left(-\mathrm{CH}\left(\mathrm{CH}_{3}\right)_{2}\right)$; Anal. Calcd for $\mathrm{C}_{24} \mathrm{H}_{22} \mathrm{O}_{4} \square 0.5 \mathrm{H}_{2} \mathrm{O}$ : C, 75.18; H, 6.05. Found: C, 75.018; H, 5.929.

Synthesis of bis(2-oxo-2H-cyclohepta[b]furan-3-yl)sulfide (10a): The solution of $\mathrm{Tf}_{2} \mathrm{O}$ (166 mg, 0.585 mmol) dissolved in $\mathrm{CH}_{2} \mathrm{Cl}_{2}(8 \mathrm{~mL})$ was added dropwise to a solution of 2a (78.9 $\left.\mathrm{mg}, 0.540 \mathrm{mmol}\right)$ and $5 \mathbf{a}$ (110 mg, $0.529 \mathrm{mmol}$ ) dissolved in $\mathrm{CH}_{2} \mathrm{Cl}_{2}(8 \mathrm{~mL})$ at $-80^{\circ} \mathrm{C}$. The suspension was warmed up to room temperature. $\mathrm{Et}_{2} \mathrm{NH}(4 \mathrm{~mL})$ was added to the solution and solvent was removed under reduced pressure. The residue was purified on silica gel column chromatography with $\mathrm{CHCl}_{3} /$ EtOAc (1:1) followed by GPC with $\mathrm{CH}_{2} \mathrm{Cl}_{2}$. Mixture of compound 10a and 4a (21.1 mg, $4.8: 1$, measured by ${ }^{1} \mathrm{H}$ NMR) was obtained. HRMS (ESI): Calcd for $\mathrm{C}_{18} \mathrm{H}_{10} \mathrm{O}_{4} \mathrm{~S}+\mathrm{Na}[\mathrm{M}+\mathrm{Na}]^{+}$345.0192. Found: 345.0191; UV/Vis $\left(\mathrm{CH}_{2} \mathrm{Cl}_{2}\right): \lambda_{\max }$, nm 258, 417; ${ }^{1} \mathrm{H}$ NMR (400 MHz, $\mathrm{CDCl}_{3}$ ): $\delta 8.21$ (bd, $1 \mathrm{H}, J=10.8 \mathrm{~Hz}, \mathrm{H}-4$ ), 7.36 (ddd, $1 \mathrm{H}, J=10.8,9.2$, $0.8 \mathrm{~Hz}, \mathrm{H}-5), 7.13$ (dd, 1H, $J=10.8,9.2 \mathrm{~Hz}, \mathrm{H}-7$ ), 7.08 (dd, 1H, $J=9.2,1.2 \mathrm{~Hz}, \mathrm{H}-8$ ), 7.01 (dddd, 1H, $J$ $=10.8,9.2,1.2,0.8 \mathrm{~Hz}, \mathrm{H}-6)$

Synthesis of bis(2-oxo-5-isopropyl-2H-cyclohepta[b]furan-3-yl)sulfide (10b): The solution of $\mathrm{Tf}_{2} \mathrm{O}$ (290 mg, $1.02 \mathrm{mmol}$ ) dissolved in $\mathrm{CH}_{2} \mathrm{Cl}_{2}(10 \mathrm{~mL})$ was added dropwise to a solution of $2 \mathbf{b}$ (185 mg, $0.983 \mathrm{mmol})$ and $5 \mathbf{b}(251 \mathrm{mg}, 1.00 \mathrm{mmol})$ dissolved in $\mathrm{CH}_{2} \mathrm{Cl}_{2}(10 \mathrm{~mL})$ at $-80{ }^{\circ} \mathrm{C}$. The suspension was 
warmed up to room temperature. $\mathrm{Et}_{3} \mathrm{~N}(4 \mathrm{~mL})$ was added to the solution and solvent was removed under reduced pressure. The residue was purified on $\mathrm{Al}_{2} \mathrm{O}_{3}$ column chromatography with $\mathrm{CH}_{2} \mathrm{Cl}_{2}$ followed by GPC with $\mathrm{CH}_{2} \mathrm{Cl}_{2}$. 10b (250 mg, $0.615 \mathrm{mmol}, 62$ \%) was obtained as yellow needle crystals.

mp 212-216 ${ }^{\circ} \mathrm{C}$; HRMS (ESI): Calcd for $\mathrm{C}_{24} \mathrm{H}_{22} \mathrm{O}_{4} \mathrm{~S}+\mathrm{Na}[\mathrm{M}+\mathrm{Na}]^{+}$429.1131. Found: 429.1130; IR (KBr disk): v $v_{\max } 3061$ (w), 2973 (w), 2955 (m), 2930 (w), 2869 (w), 1740 (s, C=O), 1593 (s), 1505 (s), 1470 (s), 1458 (s), 1420 (s), 1385 (m), 1375 (m), 1358 (m), 1320 (m), 1306 (m), 1273 (s), 1231 (s), 1115 (w), 1073 (w), 1055 (m), 1034 (m), 1005 (w), 941 (w), 920 (m), 901 (w), 882 (w), 860 (w), 801 (s), 764 (m), $756(\mathrm{~m}), 729(\mathrm{w}), 718(\mathrm{w}), 650(\mathrm{~m}), 625(\mathrm{~m}), 592(\mathrm{w}), 523(\mathrm{w}), 511(\mathrm{w}), 482(\mathrm{w}), 455(\mathrm{w}), 442(\mathrm{w})$, $423(\mathrm{w}) \mathrm{cm}^{-1}$; UV/Vis $\left(\mathrm{CH}_{2} \mathrm{Cl}_{2}\right): \lambda_{\max }, \mathrm{nm}(\log \varepsilon) 265$ (4.67), 420 (4.55); ${ }^{1} \mathrm{H}$ NMR (400 MHz, $\left.\mathrm{CDCl}_{3}\right): \delta$ 8.20 (d, $J=1.2 \mathrm{~Hz}, 2 \mathrm{H}, \mathrm{H}-4,4^{\prime}$ ), 7.07 (dd, $J=11.2,9.2 \mathrm{~Hz}, 2 \mathrm{H}, \mathrm{H}-7,7^{\prime}$ ), 6.97 (dd, $J=9.2,1.2 \mathrm{~Hz}, 2 \mathrm{H}$, H-8, 8'), 6.94 (ddd, $J=11.2,1.2,1.2 \mathrm{~Hz}, 2 \mathrm{H}, \mathrm{H}-6,6$ '), 3.01 (sept, $\left.J=6.8 \mathrm{~Hz}, 2 \mathrm{H},-\mathrm{C} \underline{\mathrm{H}}\left(\mathrm{CH}_{3}\right)_{2}\right), 1.35$ (d, $J$ $\left.=6.8 \mathrm{~Hz}, 12 \mathrm{H},-\mathrm{CH}\left(\mathrm{C}_{3}\right)_{2}\right) ;{ }^{13} \mathrm{C} \mathrm{NMR}\left(100 \mathrm{MHz}, \mathrm{CDCl}_{3}\right): \delta 169.29(\mathrm{C}-2), 159.28,158.03,154.84$, 133.88, 133.23, 127.15, 115.04, $39.73\left(-\underline{\mathrm{CH}}\left(\mathrm{CH}_{3}\right)_{2}\right)$, $23.71\left(-\mathrm{CH}\left(\underline{\mathrm{C}} \mathrm{H}_{3}\right)_{2}\right)$; Anal. Calcd for $\mathrm{C}_{24} \mathrm{H}_{22} \mathrm{O}_{4} \mathrm{~S}: \mathrm{C}$, 70.91; H, 5.46. Found: C, 70.802; H, 5.529.

\section{Synthesis of (2-oxo-2H-cyclohepta[b]furan-3-yl) (5-isopropyl-2-oxo-2H-cyclohepta[b]furan-3-yl)} sulfide (10c): The solution of $\mathrm{Tf}_{2} \mathrm{O}(175 \mathrm{mg}, 0.616 \mathrm{mmol})$ dissolved in $\mathrm{CH}_{2} \mathrm{Cl}_{2}(15 \mathrm{~mL})$ was added dropwise to a solution of 2a (74.0 mg, $0.506 \mathrm{mmol})$ and $5 \mathbf{b}(126 \mathrm{mg}, 0.503 \mathrm{mmol})$ dissolved in $\mathrm{CH}_{2} \mathrm{Cl}_{2}$ $(10 \mathrm{~mL})$ at $-80{ }^{\circ} \mathrm{C}$. The suspension was warmed up to rt. $\mathrm{Et}_{2} \mathrm{NH}(4 \mathrm{~mL})$ was added to the solution and solvent was removed under reduced pressure. The residue was purified on silica gel column chromatography with EtOAc followed by GPC with $\mathrm{CH}_{2} \mathrm{Cl}_{2}$. 10c (124 mg, $0.340 \mathrm{mmol}, 68$ \%) was obtained as yellow needle crystals.

mp 138-140 ${ }^{\circ} \mathrm{C}$; HRMS (ESI): Calcd for $\mathrm{C}_{21} \mathrm{H}_{16} \mathrm{O}_{4} \mathrm{~S}+\mathrm{Na}[\mathrm{M}+\mathrm{Na}]^{+}$387.0662. Found: 387.0661; IR (KBr disk): v $v_{\max } 3065$ (w), 3015 (w), 2961 (w), 2874 (w), 1856 (w), 1775 (s), 1752 (s), 1738 (s), 1597 (s), 1526 (m), 1507 (s), 1493 (s), 1460 (m), 1426 (w), 1412 (m), 1306 (w), 1294 (w), 1264 (m), 1231 (m), $1217(\mathrm{n}), 1057$ (w), 1048 (w), 945 (w), 928 (w), 905 (w), $885(\mathrm{w}), 864(\mathrm{w}), 855(\mathrm{w}), 804(\mathrm{~m}), 768$ (m), 754 (m), $737(\mathrm{w}), 718(\mathrm{w}), 702(\mathrm{~m}), 652(\mathrm{w}), 625(\mathrm{~m}), 486(\mathrm{w}), 450(\mathrm{w}), 438(\mathrm{w}) \mathrm{cm}^{-1}$; UV/Vis $\left(\mathrm{CH}_{2} \mathrm{Cl}_{2}\right)$ : $\lambda_{\max }, \mathrm{nm}(\log \varepsilon) 262$ (4.64), 419 (4.55); ${ }^{1} \mathrm{H}$ NMR (400 MHz, $\left.\mathrm{CDCl}_{3}\right): \delta 8.22$ (dd, $J=11.2,1.2 \mathrm{~Hz}, 1 \mathrm{H}$, H-4'), 8.19 (d, $J=1.2$ Hz, 1H, H-4), 7.34 (ddd, $J=11.2,8.8,1.2 \mathrm{~Hz}, 1 \mathrm{H}, \mathrm{H}-5$ '), 7.12 (dd, $J=10.0,9.2$ Hz, 1H, H-7'), 7.10 (dd, $J=11.2,9.2$ Hz, 1H, H-7), 7.06 (dd, $J=9.2,1.2$ Hz, 1H, H-8'), 7.00 (dd, $J=9.2$, $0.8 \mathrm{~Hz}, 1 \mathrm{H}, \mathrm{H}-8$ ), 7.00 (dddd, $J=10.0,8.8,1.2,1.2 \mathrm{~Hz}, 1 \mathrm{H}, \mathrm{H}-6$ '), 6.97 (ddd, $J=11.2,1.2,0.8 \mathrm{~Hz}, 1 \mathrm{H}$, H-6), 3.02 (sept, $\left.J=6.8 \mathrm{~Hz}, 1 \mathrm{H},-\mathrm{C} \underline{\mathrm{H}}\left(\mathrm{CH}_{3}\right)_{2}\right), 1.36$ (d, $\left.J=6.8 \mathrm{~Hz}, 6 \mathrm{H},-\mathrm{CH}\left(\mathrm{C}_{3}\right)_{2}\right) ;{ }^{13} \mathrm{C} \mathrm{NMR}(100 \mathrm{MHz}$, $\left.\mathrm{CDCl}_{3}\right): \delta$ 169.23, 168.94, 159.32, 158.31, 157.94, 155.03, 154.88, 137.19, 134.00, 133.79, 133.30, 132.48, 130.04, 127.17, 116.00, 115.24, 101.24, 98.99, $39.73\left(-\underline{\mathrm{CH}}\left(\mathrm{CH}_{3}\right)_{2}\right), 23.71\left(-\mathrm{CH}\left(\underline{\mathrm{C}} \mathrm{H}_{3}\right)_{2}\right)$; Anal. Calcd for $\mathrm{C}_{21} \mathrm{H}_{16} \mathrm{O}_{4} \mathrm{~S} \square 0.25 \mathrm{H}_{2} \mathrm{O}$ : C, 68.37; H, 4.51. Found: C, 68.552; H, 4.663. 


\section{REFERENCES}

1. a) J. B. Hendrickson and S. M. Schwartzman, Tetrahedron Lett., 1975, 16, 273. b) V. G. Nenajenko, P. V. Vertelezkij, I. D. Gridnev, N. E. Shevchenko, and E. S. Balenkova, Tetrahedron, 1997, 53, 8173. c) I. L. Baraznenk, V. G. Nenajdenko, and E. S. Balenkova, Tetrahedron, 2000, 56, 3077.

2. a) S. Seto, Sci. Rep. Tohoku University, First Series, 1953, 37, 367. b) T. Nozoe, S. Seto. S. Matsumura, and T. Terasawa, Chem. \& Ind., 1954, 1356. c) T. Sato, “Bulletin of the Chemical Research Institute of Non-Aqueous Solution” (Tohoku University) 1959, 8, 47. d) N. Morita, M. Kudo, R. Yokoyama, and S. Ito, Heterocycles, 2001, 54, 679.

3. A. G. Anderson Jr. and J. A. Nelson, J. Am. Chem. Soc., 1950, 72, 4980. b) K. Hafner, A. Stephan and C. Benhard, Liebigs Ann. Chem., 1958, 625, 108. c) K. Hafner, A. Stephan, and C. Benhard, Liebigs Ann. Chem., 1961, 650, 42. d) R. N. McDonald, R. R. Reitz, and J. M. Richmond, J. Org. Chem., 1976, 41, 1822.

4. a) S. Naya and M. Nitta, J. Chem. Soc., Perkin Trans. 2, 2000, 2427. b) S. Naya and M. Nitta, J. Chem. Soc., Perkin Trans. 1, 2000, 2777. c) S. Naya, T. Sakakibara, and M. Nitta, J. Chem. Soc., Perkin Trans. 2, 2001, 1032. d) S. Naya, M. Isobe, Y. Hano, and M. Nitta, J. Chem. Soc., Perkin Trans. 2, 2001, 2253. e) S. Naya and M. Nitta, Tetrahedron, 2003, 59, 4157. f) S. Naya, K. Yoda, and M. Nitta, Tetrahedron, 2004, 60, 4953. g) S. Naya, K. Yoda, and M. Nitta, Tetrahedron, 2005, 61, 6816.

5. N. Morita, T. Matsuki, M. Nakashima, T. Shoji, K. Toyota, S. Kikuchi, and S. Ito, Heterocycles, 2006, 69, 119.

6. N. Morita, J. Higashi, K. Okada, T. Shoji, K. Toyota, M. Watanabe, M. Yasunami, S. Kikuchi, and S. Ito, Heterocycles, 2007, 73, 237.

7. T. Shoji, J. Higashi, S. Ito, K. Toyota, T. Asao, M. Yasunami, K. Fujimori, and N. Morita, Eur. J. Org. Chem., 2008, 1242.

8. A. Thompson, R. J. Butler, M. N. Grundy, A. B. E. Laltoo, K. N. Robertson, and T. S. Cameron, J. Org, Chem., 2005, 70, 3753. 